The case of sulfonation in the chemical synthesis of oligonucleotides

\title{
A.Kraszewski, J.Stawiński and M.Wiewiórowski*
}

Department of Stereochemistry of Natural Products, Institute of Organic Chemistry, Polish Academy of Sciences, Noskowskiego 12/14, 61-704 Poznan, Poland

Received 5 March 1980

\section{ABSTRACT}

The sulfonation of nucleosidic component, a side reaction during phosphotriester bond formation, as a function of the reactivity of the condensing agents and the kind of substituents in the starting phosphodiester is discussed. It was found that in the coupling reaction of nucleoside alkyl phosphodiesters, the degree of sulfonation of the nucleosidic component was very high; while under the same conditions when the aryl group was present in the corresponding phosphodiester, this side reaction practically did not occur.

\section{INTRODUCTION}

Since 1966 when Khorana introduced arylsulfonyl chlorides into nucleotide chemistry, they have become commonly employed as reagents for the activation of phosphomono- and phosphodi-esters in the synthesis of oligonucleotides ${ }^{1-3}$. The major drawback of these reagents, however, is that besides the activation of phosphoric acid esters, they also caused extensive sulfonation of the nucleosidic component 1,2 . This last reaction can be considerably suppressed when sterically hindered arylsulfonyl chlorides $^{1}$ are used or chlorides are replaced by azolides (better leaving groups ${ }^{2,3,4}$ ).

During our studies on oligothymidylic acid synthesis using alkyl esters of glycolic acid as an internucleotide phosphate protective group 5 and 2, 4, 6-triisopropylbenzenesulfonyl tetrazole (TPS-Te) as a condensing reagent ${ }^{3}$, we have noticed a high degree of sulfonation (ca 20\%) which makes synthesis of oligomers higher than octamer very difficult. These results were quite unexpected because TPS-Te has been until now known as a coupling agent with low ability for sulfonation in condensation reaction ${ }^{6,7}$. These results prompted us to take a closer look at the condensation reaction, and at the coupling agents and to consider the additional factor which may influence the extent of sulfonation, namely the kind of phosphate protective groups in phosphodiesters.

Being interested mainly in synthetic implications of this research, we chose for our studies the representative phosphodiesters being currently used in the chemical synthesis of oligonucleotides (2-cyanoethyl, 2,2,2-trichloroethyl, p-chlorophenyl nucleoside phosphodiesters), or those being under investigation in this laboratory (benzyl glycolate nucleoside phosphodiester). Nucleoside ethyl phosphodiester, although it has no synthetic application in oligonucleotide chemistry, was used as a reference compound for our studies. As a condensing reagent, only two representative compounds were applied, i. e., benzenesulfonyl triazole ${ }^{2}$ (BST) as a mild activating reagent and TPS-Te as a powerful condensing agent. 


\section{RESULTS_AND DISC}

We tried to correlate the unexpected high degree of sulfonation when esters of glycolic acid were used as phosphate protective groups with the significantly lower reactivity of nucleoside alkyl phosphodiesters in condensation reaction as comparing to analogous reaction with nucleoside aryl phosphodiesters. These differences in the rates of condensation, in our opinion, are closely connected with the different inductive effects of alkyl and aryl substituents which lead to the formation of a different partial positive charge on phosphorous atom. This in turn may influence the rate of activation of phosphodiester or the reactivity of active phosphorylating species formed from corresponding phosphodiester in the presence of condensing agents ${ }^{8}$. To check this hypothesis we prepared five phosphodiesters Ia - Ie with aryl and different alkyl groups and condensed them with nucleoside II in the presence of TPS-Te. After work-up (see Experimental Section) all reaction mixtures were chromatographed on silica gel columns and the results are shown in Table 1. As can be seen, the time of the condensations when nucleoside alkyl phosphodiesters were used (reactions $1-4$ in Table 1) is ten times longer than in analogous reaction for nucleoside aryl phosphodiester (reaction 5). Also the degree of sulfonation is much higher for nucleoside alkyl than for nucleoside aryl phosphodiesters and decreases when the alkyl substituents become less electron-donating.

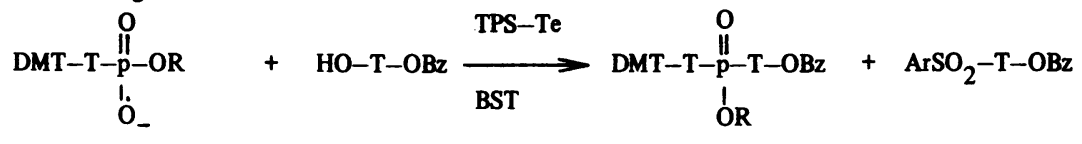

I
II
III

$$
\begin{array}{ll}
\text { IIIa } & R=\mathrm{CH}_{3} \mathrm{CH}_{2}- \\
\text { IIIb } & R=\mathrm{PhCH}_{2} \mathrm{OCOCH}_{2}- \\
\text { IIIc } & R=\mathrm{NCCH}_{2} \mathrm{CH}_{2}- \\
\text { IIId } & R=\mathrm{Cl}_{3} \mathrm{CCH}_{2-} \\
\text { IIIe } & R=\mathrm{p}-\mathrm{ClPh}_{-}-
\end{array}
$$

\begin{tabular}{|c|c|c|c|c|c|c|}
\hline \multirow{2}{*}{$\begin{array}{l}\text { Reaction } \\
\text { No. }\end{array}$} & \multirow[t]{2}{*}{ Phosphodiester I } & \multirow{2}{*}{$\begin{array}{c}\text { Coupling } \\
\text { agent }\end{array}$} & \multirow{2}{*}{$\begin{array}{l}\text { Time } \\
\text { (hrs) }\end{array}$} & \multicolumn{3}{|c|}{ Isolated yields of } \\
\hline & & & & III & Il & IV \\
\hline 1. & $\mathrm{R}=\mathrm{CH}_{3} \mathrm{CH}_{2}-$ & TPS-Te & 20 & $60 \%$ & $10 \%$ & $27 \%$ \\
\hline 2. & $\mathrm{R}=\mathrm{PhCH}_{2} \mathrm{OCOCH}_{2}-$ & TPS-Te & 20 & $60 \%$ & $10 \%$ & $17 \%$ \\
\hline 3. & $\mathrm{R}=\mathrm{NCCH}_{2} \mathrm{CH}_{2}-$ & TPS-Te & 20 & $50 \%$ & $15 \%$ & $13 \%$ \\
\hline 4. & $\mathrm{R}=\mathrm{Cl}_{3} \mathrm{CCH}_{2}^{-}$ & TPS-Te & 20 & $80 \%$ & $8 \%$ & $8 \%$ \\
\hline 5. & $\mathrm{R}=\mathrm{p}-\mathrm{ClPh}-$ & TPS-Te & 2 & $95 \%$ & $0 \%$ & traces \\
\hline 6. & $\mathrm{R}=\mathrm{PhCH}_{2} \mathrm{OCOCH}_{2}-$ & BST & 96 & $60 \%$ & $40 \%$ & traces \\
\hline
\end{tabular}

Table 1. Composition of reaction mixtures after condensations*

* For reaction condition see Experimental Section. 
The use of the stoichiometric or larger amounts (3-4 eqv.) of the condensing reagent did not affect substantially the rate of condensation. This seems to suggest that the lower rate of the internucleotide bond formation in case of nucleoside alkyl phosphodiesters was caused by the lower reactivity of the phosphorus atom in the active phosphorylating species?.

The sulfonation of the 5 hydroxyl group in the nucleosidic component II during condensation can occur in two main ways: (i) direct sulfonation by a condensing agent, and or (ii) sulfonation via mixed phospho-sulfonic anhydride.

To evaluate the participation of each of these routes in the total sulfonation, we carried out the condensation of nucleoside alkyl phosphodiester Ib with the nucleoside II in the presence of BST, which is known to have very weak sulfonating properties ${ }^{2}$. Assuming that the activation of phosphodiesters by BST and TPS-Te proceeds via a similar mixed anhydride, and if they are active sulfonating species, the degree of sulfonation in the presence of BST should be at least the same as when TPS-Te was used ${ }^{10}$. As can be seen from Table 1 (reaction 6) during condensation in the presence of BST only traces of a sulfonated product were formed. This strongly suggests that sulfonation occurs according to route (i) i. e., by direct sulfonation with a condensing agent. Hence, also, in the first four reactions in Table 1, the observed sulfonation has to be attributed to direct sulfonation by TPS-Te. These findings also indicate that TPS-Te has rather strong sulfonylating properties. On the basis of our additional experiments (results not snown) we have found that, if sulfonation is the only possible reaction pathway of this condensing agent, the sulfonating properties of TPS-Te are indeed similar to those of TPS ${ }^{11}$. However, during condensation, when two parallel reactions are possible, i. e., condensation and sulfonation, TPS-Te exhibits significantly lower ability for sulfonation than TPS.

From the above it is clear that evaluation of the utility of the aryl sulfonic acids derivatives as coupling reagents can not be done by simple comparision of their sulfonating properties in model experiments (sulfonation of nucleoside). In our opinion the most important factors are the rate of condensation and the relative ratio of the rates of condensation and sufonation. The first factor reflects the time of the condensation and the second one, the degree of sulfonation occuring during condensation.

When one applies these criteria to the results from Table 1 the following conclusions can be drawn: During coupling of nucleoside aryl phosphodiester with nucleoside in the presence of TPS-Te (reaction 5), the condensation reaction is very fast, so the ratio of the rates of condensation and sulfonation is also high. In the case of nucleoside alkyl phosphodiesters (reactions $1-4$ ) this ratio is much lower because of a considerably slower condensation reaction while the rate of sulfonation is still the same as for nucleoside aryl phosphodiesters. As a result, for nucleoside aryl phosphodiester a very low sulfonation was observed, while for the corresponding alkyl phosphodiester the degree of sulfonation was very high.

When BST was used as a condensing agent, the high ratio of rates of condensation and sulfonation was the reason why only traces of a sulfonated product were observed. On the other hand, the very low rate of condensation (after four days $40 \%$ of unreacted compound II was present) makes very questionable the synthetic application of this coupling reagent for the activation of nucleoside alkyl phosphodiester.

These results seem to suggest that the alkyl groups are not suitable for the protection of the internucleotidic bonds. For these phosphodiesters, the low rates of condensation favour sulfonation when a powerful condensing agent is used, whereas milder condensing agent, although suppressing the sulfonation, are not reactive enough to complete the reaction in reasonable time. Thus the efforts in searching for new internucleotidic phosphate protective groups, in our opinion, should be concentrated on aryl rather than on alkyl groups, at least when derivatives of aryl sulfonic acids are used as the activating reagents. 


\section{EXPERIMENTAL SECTION}

\section{MATERIALS AND METHODS}

TPS-Te ${ }^{3}$ and $\mathrm{BST}^{2}$ were prepared according to the published procedures. TLC analysis was performed on Kieselgel $60 \mathrm{~F}_{254}$ plates (Merck) in solvent A: chloroform-methanol $(9: 1 \mathrm{v} / \mathrm{v})$ and solvent B: chloroform-methanol (8:2 v/v ). Short column chromatography ${ }^{12}$ on TLC Kieselgel type H (Merck) in chloroform containing methanol $(0-4 \%)$ was used for separation of phosphotriesters.

\section{GENERAL PROCEDURE FOR CONDENSATION}

Phosphodiester I $(0.36 \mathrm{mM})$ and nucleoside II $(0.30 \mathrm{mM})$ were dissolved in pyridine and rendered anhydrous by repeated evaporation of added pyridine, in a vacuum, each time the flask being opened in a dry atmosphere of nitrogen. After addition of the coupling agent (TPS-Te or BST, $0.72 \mathrm{mM}$ ) reaction mixture was concentrated to ca $3 \mathrm{ml}$ volume and kept closed for period of time as listed in Table 1. The reaction mixture was then decomposed by addition of water, concentrated to a gum and, after dissolving in chloroform, washed with $1.0 \mathrm{M}$ phosphate buffer $(\mathrm{pH} 7.5)$. The organic layer was separated, dried over anhydrous sodium sulphate, evaporated and chromatographed on a silica-gel short column in chloroform containing $0-4 \%$ methanol.

\section{ANALYSIS}

The purity of all isolated fully protected dinucleoside monophosphates was checked by chromatography on silica-gel plates before and after removal of the dimetoxytrityl groups, and the structure was proved by ${ }^{1} \mathrm{H}$ NMR analysis. Sulfonated products were characterized by ${ }^{1} \mathbf{H}$ NMR spectra and compared with compounds obtained by direct sulfonation of II with TPS-Te and BST.

The chromatographic mobilities in solvent $\mathbf{A}$ for the isolated fully protected dinucleoside monophosphates IIIa - IIIe and sulfonated products with respect to $3^{\circ}-0$ - benzoylthymidine are as follow:

$$
\begin{aligned}
& \text { IIIa - 1.20; IIIb - 1.26; IIIc }-1.00 \text {; IIId }-1.22 \text {; } \\
& \text { IIIe - 1.29; and for TPS-0-T }-0-B z 1.75 ; \mathrm{BS}-0-\mathrm{T}-0-\mathrm{Bz}-1.52
\end{aligned}
$$

\section{ACKNOWLEDGEMENT}

This work was supported by the Polish Academy of Sciences, project W- 09. 7. 2. 3. 1 .

\section{REFERENCES}

1. Lohrman, R. and Khorana, H. G. (1966) J._Am. Chem. Soc. 88, 829

2. Katagiri, N., Itakura. K. and Narang. S. A. ibid. (1975) 97, 7332

3. Stawiński, J., Hozumi, T. and Narang, S. A. (1976) Can._J._Chem. 54, 670

4. Berlin, Yu. A., Chakhmakhcheva, O. G., Efimov, V. A., Kolosov, M. N. and Korobko, Y. G. (1973) Tetrahedron_LEte.t. 1353

5. Kraszewski, A., Stawiński, J. and Wiewiórowski, M. - in preparation.

6. Stawiński, J., Hozumi, T., Narang, S. A., Bahl, C. P. and Wu, R. (1977) - Nucleic_Acids_Res.4,353,

7. Hirose, T., Crea, R. and Itakura, K. (1978) Tetrahedron_Lett. 2049 
8. Inductive effects of the substituents have been found to have a great influence on the reactivity of tetra-substituted pyrophosphates which may be an active phosphorylating species in condensation reactions. See Knorre, D. G. and Zarytova, V. F. Proceedings of the International Conference „Recent Developments in the Chemistry of $t-R N A$ and Minor Bases“, Poznari, 1974.

9. If the lower rates of condensation would be caused by slower activation of phosphodiesters, a larger excess of condensing agent should accelerate this reaction. To obtain more convincing evidence, ${ }^{31} P$ NMR studies are needed.

10. Phospho-benzenesulfonic anhydride should exhibit even higher reactivity than analogous anhydride containing 2,4,6- triisopropyl-benzenesulfonyl group. BST was used since 2,4,6-triisopropylbenzenesulfonyl triazole is not reactive enough in the condensation of nucleoside alkyl phosphodiester with nucleoside.

11. Nucleoside II was treated with two eqv. of TPS or TPS-Te in pyridine $(3 \mathrm{ml}$ for $1 \mathrm{mM}$ of nucleoside II) and the degree of sulfonation was estimated after inspection of silica-gel plates under UV lamp.

12. Hunt, B. J. and Rigby, B. (1967) Chem. Ind. (London) 1868. 